\title{
Healthcare Preferences Among Lesbians: A Focus Group Analysis
}

\author{
MARGARET R. SEAVER, M.D., M.P.H., ${ }^{1,2,3}$ KAREN M. FREUND, M.D., M.P.H., ${ }^{2,3}$ \\ LESLIE M. WRIGHT, M.P.H., ${ }^{2,3}$ JENNIFER TJIA, M.D., M.S.C.E., ${ }^{4}$ \\ and SUSAN M. FRAYNE, M.D., M.P.H. $5,6,7$
}

\begin{abstract}
Objective: The healthcare needs of lesbians are not well understood. We sought to characterize lesbians' experiences with, and preferences for, women's healthcare.

Methods: We conducted three age-stratified focus groups (18-29, 30-50, and $>50$ years) with a total of 22 participants using a semistructured interview guide to elicit lesbians' experiences and preferences. We analyzed transcripts of these audiotaped sessions using the constant comparative method of grounded theory. Community-dwelling women who self-identified as lesbian and responded to advertisements were selected on first-come basis.

Results: Participants voiced experiences and preferences for healthcare that emerged into three themes: desired models of care, desired processes of care, and desired patient-provider relationship. Each theme was further developed into multiple subthemes. Within the subthemes we identified issues that were specific to lesbians and those that were general women's health issues. Participants preferred, but did not always receive, care that is comprehensive in scope, person centered, nondiscriminatory, and inclusive of them as lesbians.

Conclusions: Healthcare providers, institutions, and society should adopt an inviting, personcentered approach toward lesbians seeking healthcare, assure them access to healthcare information, and establish healthcare delivery systems that take all aspects of health into account.
\end{abstract}

\section{INTRODUCTION}

L ESBIANS ARE AN IMPORTANT and underrecognized patient population about which little is known. The Institute of Medicine (IOM) has specif- ically addressed the need for research in this population by stating that studies are needed "[to] identify possible barriers to mental and physical healthcare services [for lesbians] and ways to increase their access to these services."1(p10) Without

\footnotetext{
${ }^{1}$ VA Boston Healthcare System, Boston, Massachusetts.

${ }^{2}$ National Center of Excellence in Women's Health, Boston University School of Medicine, Boston, Massachusetts.

${ }^{3}$ Women's Health Unit, Section of General Internal Medicine, Department of Medicine, Boston University Medical Center, Boston, Massachusetts.

${ }^{4}$ Division of Geriatric Medicine, Department of Medicine, University of Pennsylvania, Philadelphia, Pennsylvania.

${ }^{5}$ Center for Health Care Evaluation, VA Palo Alto Health Care System, Palo Alto, California.

${ }^{6}$ Division of General Internal Medicine, and ${ }^{7}$ Center for Primary Care and Outcomes Research, Stanford University School of Medicine, Palo Alto, California.

Support for this research was provided by contracts from the U.S. Public Health Service, Office of Women's Health, and by the U.S. Department of Veterans Affairs and Health Services Research and Development Service grant RCD 98-312.

The views expressed in this paper are those of the authors and do not necessarily represent the views of the Department of Veterans Affairs.
} 
data describing the healthcare needs of lesbians, clinicians will be unable to design interventions to improve the quality of care for this group of patients who comprise $3 \%-10 \%$ of all U.S. women. ${ }^{1-3}$

Unfortunately, the literature base identifying optimal approaches to the delivery of lesbian health care is thin. The available literature supports the assertion that lesbians use healthcare less than heterosexual women do and that they may experience lower health status because of this. ${ }^{1,4-7}$ For example, studies have documented lower rates of breast and cervical cancer screening among lesbians. ${ }^{1,8}$ Other studies have focused on health behaviors, such as smoking and alcohol consumption, and have found that lesbians use these substances, which are closely linked to poor health outcomes, more than heterosexual women do. ${ }^{7,9-11}$ Although some authors have pointed to access problems (e.g., lack of insurance) as key barriers to healthcare, ${ }^{4,6,7}$ others have concluded that negative experiences with the healthcare system promote avoidance of care. ${ }^{9,12,13}$ To inform interventions designed to address such disparities, more information about the healthcare preferences of lesbians is needed.

Prior studies on lesbian health have been limited by several methodological challenges. First, it is difficult to define who is a lesbian, and definitions differ between studies. The term "lesbian" can refer to sexual behavior (i.e., women who have sex with women, although not necessarily exclusively with women), sexual orientation (i.e., women who have a sexual interest in other women, regardless of whether they have sex with other women), and cultural identity (i.e., women who identify with a community of likeminded women). ${ }^{14}$ This variation may lead to potential problems in generalizability and precision of cohort definitions. Second, lesbians are a heterogeneous group belonging to every age group, ethnic group, and socioeconomic class. ${ }^{1}$ Demographic cohort selection effects may influence assessments of healthcare needs. Third, it can be difficult to identify and recruit potential research participants. Some early lesbian health studies recruited women from bars and nightclubs, limiting the generalizability of findings to women who were often smokers and alcohol users. ${ }^{7}$ Often marginalized and subjected to social stigma, ${ }^{15}$ lesbians recruited in other settings may hesitate to disclose their sexual orientation to investiga- tors. ${ }^{12,16}$ Healthcare settings, which are usually an excellent site for research participant recruitment, may be less helpful for the recruitment of lesbians because healthcare providers typically are unaware of their patients' sexual orientations. ${ }^{17}$ Thus, for various reasons, subgroups of lesbians may not enroll in studies recruiting from healthcare settings, potentially introducing bias.

To address the call for a better understanding of lesbians' healthcare needs and attempt to address these methodological difficulties, we recruited self-identified lesbians from the community and conducted an exploratory study using focus groups. The specific aim of this study was to characterize lesbians' experiences with and expectations of women's healthcare. To our knowledge, the structure of healthcare preferred by lesbians has not been examined previously.

\section{MATERIALS AND METHODS}

\section{Participants and recruitment}

Women were eligible to participate in this study if they were aged $\geq 18$, community dwelling, and self-identified as lesbians. Potential participants were recruited using advertisements in newspapers serving the gay and lesbian community and fliers posted at Boston area businesses and primary care practices. Three age-stratified groups $(18-29,30-50,>50$ years) of up to 9 participants each were enrolled on a first-come basis. Participants were age stratified so that discussion would benefit from both their shared experience of time and world events and their shared age-based healthcare needs. ${ }^{18,19}$ Participants received an honorarium. The study was approved by the Institutional Review Board at the Boston University School of Medicine.

\section{Instruments}

The 27-item, semistructured focus group protocol used in this study was developed jointly by six sites of the Department of Health and Human Services (DHHS) National Centers of Excellence in Women's Health. ${ }^{20}$ Drawing on a literature review and expert input, questions on the protocol were designed to elicit knowledge, attitudes, and beliefs about women's health, the healthcare environment, and the participants' own experience with the healthcare system. The focus group for- 
mat, which uses an inductive approach, has been advocated for the study of topics like this that have received little prior investigation. ${ }^{21}$ Participants also completed a brief demographic questionnaire.

\section{Focus groups}

In separate arms of the larger National Centers of Excellence in Women's Health study, other sites applied the same protocol to different study populations. ${ }^{22}$ We report the results of the study arm that enrolled lesbians. The focus groups were conducted in a private conference room in a major medical center by a single interviewer with expertise in qualitative research methods. The interviewer facilitated discussion, assured that all participants had an opportunity to participate, and asked for clarification or elaboration if needed but did not direct the content of participants' comments. One or two other investigators observed each focus group. Each 2.5-hour session was audiotaped and then transcribed verbatim.

\section{Analysis}

After checking of transcriptions for accuracy of content, the transcripts were analyzed using the constant comparative method of grounded theory. ${ }^{23,24}$ In this method, the analytical process undergoes continuous refinement, repeatedly feeding back into the process of coding and iteratively honing the identified themes. Five independent reviewers analyzed transcripts in four stages. In the first stage, the five reviewers read each transcript identifying key words and phrases that represented an idea or concept expressed by the participants. Key words and phrases were in the participants' own words whenever possible. In the second stage, reviewers met to discuss their initial key words and phrase codes. When key words and phrases demonstrated a clear relationship to each other, reviewers aggregated these related ideas into themes, such as patientprovider relationship, and subthemes within each theme. In the third stage, reviewers recoded the transcripts, applying the newly developed themes and subthemes to each transcript. At this stage, transcripts were further coded according to whether the expressed ideas were specific to lesbians or applied to women's health in general. In the fourth and final stage of analysis, reviewers met to identify and review differences in coding.
Differences were reconciled through consensus. Throughout this process, the reviewers repeatedly returned to the original transcripts to assure that our analysis remained grounded in the participants' ideas. In this way indigenous themes that characterized the experience of the participants were identified. ${ }^{25}$ Analysis was facilitated by the use of the qualitative software package NUD*IST V4.0 (Qualitative Solutions and Research, Melbourne, Australia).

\section{RESULTS}

Twenty-two women who self-identified as lesbians participated (groups of 8, 9, and 5 in the youngest, middle, and oldest age groups, respectively). The mean age was 38 (range 22-63). Nine were from racial/ethnic minority groups. Twenty reported having had a healthcare visit within the past year, and 10 reported having made more than four healthcare visits in the past year. Five had no regular healthcare provider, 4 had no health insurance, 4 had less than a college degree, and 9 had household income $<\$ 35,000$ (2 did not provide income information).

We identified three major themes: model of care, process of care, and the patient-provider relationship, and several subthemes within each theme. We describe each theme and subtheme, providing representative quotations to illustrate these themes and dissenting views when present. ${ }^{26}$

Our analysis also identified several key preferences that were specific to lesbians (Table 1). Within the theme of model of care, women preferred comprehensive care that was inclusive of lesbians, provided by clinicians who had specific knowledge about lesbians and who were able to provide health information specifically about lesbians. Within the theme of process of care, women's preferences included sufficient time to disclose information about their sexuality and assurance that their sexual orientation would be treated confidentially. Participants' preference was for settings where there were lesbian-specific materials in the waiting room and sexual orientation options on the intake forms. Within the theme of patient-provider relationship, the preference was for a patient-provider relationship that encouraged disclosure of sexual orientation (promoted by providers who are nonheterosexist 
Table 1. Themes and Key Issues Specific to Lesbians

\begin{tabular}{|c|c|}
\hline Themes and subthemes & Sample key issues specific to lesbians \\
\hline \multicolumn{2}{|l|}{ Model of care } \\
\hline Comprehensive care & Inclusive of lesbians \\
\hline Incorporation of integrative health & Specific health information for lesbians \\
\hline Access to health information & Specific training for providers about lesbians \\
\hline Women's health training for providers & \\
\hline \multicolumn{2}{|l|}{ Process of care } \\
\hline Time constraints & Sufficient time with providers to disclose and discuss \\
\hline Payment systems & sexual orientation \\
\hline \multirow[t]{3}{*}{ Office systems } & Confidentiality especially about sexual orientation \\
\hline & Lesbian-specific information in waiting rooms \\
\hline & Sexual orientation options on intake forms \\
\hline \multicolumn{2}{|l|}{ Patient-provider relationship } \\
\hline Communication style & Open communication to enhance opportunities for \\
\hline Provider characteristics & disclosure and discussion of sexual orientation \\
\hline \multirow[t]{2}{*}{ Patients being known as individuals } & Nonheterosexist, nonjudgmental providers \\
\hline & $\begin{array}{l}\text { Providers attuned to lesbian-specific concerns of their } \\
\text { patients }\end{array}$ \\
\hline
\end{tabular}

and nonjudgmental) and for a provider attuned to the specific concerns of lesbians.

\section{Model of care}

Model of care was one of three major themes that emerged from the analysis. Comments were categorized as model of care if they related to an overarching philosophy of healthcare delivery and the scope of care. Four subthemes were identified: (1) comprehensive care, emphasizing preventive care and mental health, (2) incorporation of integrative health into routine care, (3) access to health information, and (4) special women's health training for providers.

Comprehensive care. Some participants identified the ideal model of care as comprehensive care. This was defined as care that addressed the whole person in an integrated fashion and that was not limited to gynecological or reproductive care. This applied to acute care, preventive care, nutrition, and mental healthcare: "Sometimes, in mainstream society, when people think 'women's health' that necessarily means reproductive health and not comprehensive healthcare, onestop shopping." Mental healthcare was identified as an important aspect of a comprehensive model of care: "My healthcare has kind of been a little separated. It's like there is mental health and then there's 'real' health. That's the way it's been presented."

Participants also identified barriers to delivering this model of women's healthcare. One idea that emerged was that the traditional definition of women's health focused on reproductive health, a paradigm that hinders the acceptance of a more comprehensive model. Another obstacle described is the use of separate clinics to deliver different types of care (e.g., urgent, preventive, reproductive, mental healthcare, as well as chronic disease management): "You go to a primary care physician, and then, do you go to a separate gynecologist? Then say you have heart trouble, do you go to a separate cardiologist? Yes, you do all those things."

Participants believed that women's health, as it is usually practiced without this comprehensive model, was not inclusive of lesbians. Most had felt marginalized by a healthcare system that emphasized reproductive health and implicitly assumed that all patients are heterosexual: "Every time I went to a gynecologist-that was about the only time I went to a doctor- $\mathrm{I}^{\prime} \mathrm{d}$ be the only person in the room who wasn't pregnant, and I felt I'm going to be the last person waited on here because they're never going to make any money on me; I'm not going to have any children."

The groups expressed the idea that lesbians felt excluded from healthcare. The emphasis on reproductive health gave the impression that lesbians do not need gynecological examinations. The marginalization they experienced extended to their families. Participants stated that partners were often excluded from the healthcare process in instances where a heterosexual partner would have been included. One participant had had her partner included in her healthcare: "They let my partner come in with me. They treated her nor- 
mally. It was such a joy to think they had taken the time to think that I wasn't widowed or divorced and that I did have a partner and that I could include this person in my follow-up meetings."

Incorporating Integrative Health. The second subtheme expressed by the participants was the importance of including integrative health into traditional healthcare. Most participants used some form of integrative therapy, including herbal medicine, acupuncture, vitamins, massage, meditation, and chiropractors. They wanted their mainstream practitioners (doctors and nurses) to know about these practices and incorporate them into treatment plans. The general concept of integrative health appeared to be a fundamental component of most participants' conceptualizations of healthcare: "I really want it to be a place that's very integrated, where I can feel like I can bring my whole self, I can bring my questions about my yeast infections, I can bring my questions about this Chinese drug." Other participants echoed this sentiment: "And you don't have to worry what the doctor at the acupuncture center is telling you, or the herbal therapist is telling you, and then what the Western doctor is saying, that there would be integration of those aspects as well."

However, participants understood the traditional model of care as exclusive of integrative therapies: "When I first started seeing a chiropractor, I was seeing a doctor who had no use for chiropractors at all, and they would get into this back and forth, debasing each other, and telling me it was stupid to see the other person." In addition to perceived conflict between providers, a lack of insurance coverage and lack of referral sources were identified as barriers to a comprehensive model of care.

Access to Information. The third major subtheme defining model of care was access to information about health, obtained from books, the Internet, and providers. Some women (especially in the older group) commented that information about sexual development and sexual identity was difficult to obtain from any source. This served as a barrier to ideal care: “It's her body, but don't tell her about it. You had to find out [about sexual development] on the street, from your friends." Participants identified the need for more explanation of diagnoses, treatment, and procedures, especially gynecological and surgical procedures, from healthcare providers: "There were two gynecologists; I wasn't feeling that they were giving me information."

One participant stated that fliers in waiting rooms could provide the opportunity for further discussion of health issues with practitioners. Participants thought that lay sources were also good sources of information. Women identified support and informational groups as currently underused, suggesting that groups could enhance access to information on such topics as stress, menopause, and reproductive health.

Participants identified language as a barrier to exchange of information with providers. Misinformation-from the Internet, family, friends, "the street," and even unknowledgeable providers-was another barrier to good healthcare: "There's so much terrible stuff on the web. It can be a source of extremely bad, wrong, kind of information."

Participants stated that health information specific to lesbians was particularly difficult to access. Waiting rooms often lack lesbian-specific information. Providers often lack information about lesbian health, such as their risks of HIV or other sexually transmitted diseases (STDs), and their risk of breast and gynecological cancers: "I'm with a partner, and have been for two years, who's HIV positive. I'm always going to have questions. I'm concerned about different types of sex." "I've never seen any mention of a dental dam or rubber gloves in a doctor's office."

However, some women did experience women's health in specific settings as inclusive and supportive of them as lesbians. These settings included clinics devoted to the care of gay men and lesbians and offices where healthcare providers identified themselves as lesbian or gay, or advertised (by word of mouth) that they were oriented to the care of lesbians. Women perceived providers who ask about sexual orientation as more open to lesbians.

Specialized knowledge of women's health. The fourth major subtheme in the models of care theme, closely related to the need for access to information, was the importance of providers having specialized knowledge in women's health and lesbian health. Participants identified lack of specialized training as a barrier to healthcare for women in general and especially lesbians and recommended that medical school curricula fo- 
cus on lesbian health, lesbian lifestyle or culture, and integrated healthcare approaches: "How many providers know about safe lesbian sex?" "Women who haven't had children, are they more or less likely to have breast cancer? There's a different constellation of health issues that are part of the lesbian community."

\section{Process of care}

Process of care was the second major theme that emerged from the analysis. Comments were categorized as process of care if they related to systems issues in the delivery of healthcare. Three major subthemes were (1) time constraints as they impacted both the patient and the provider, (2) payment systems including insurance and managed care, and (3) office systems including confidentiality, comfort in the office, and referral.

Time constraints. The first subtheme, time constraints, included limited time for appointments that impeded getting/giving a complete history and adversely affected quality of care. Providers strapped for time might not review a patient's history prior to a visit, leading to unnecessary testing: "Every time I've had a Pap smear, they have called on the phone and told me to come back and take it again, that it was no good. I tell them that I've had a hysterectomy, and they say 'Oh you did? Well, then okay.' But it scares you, when that call comes in."

Women reported that ideal care would leave them feeling like people as opposed to numbers. Insufficient time to review their history and social issues with providers led women to the feel that they were widgets on an assembly line. Women also believed that their time was as valuable as the practitioner's, and, therefore, they did not like to wait or reschedule. They equated respect for their time with respect for them as people.

Payment systems. The second major subtheme in process of care related to payment systems, including insurance issues and managed care. Most participants appreciated getting their care at a single location, both for convenience and to reduce the stress of visits to multiple providers. They wanted more information about navigating the increasingly complex medical system. They also wanted their providers to communicate with each other.

Participants believed that healthcare was diffi- cult to access especially if they had no insurance and that this led to inappropriate emergency room use or delaying and avoiding healthcare. Incomplete coverage for comprehensive care was an additional concern. Participants wondered why integrative care, such as acupuncture and massage, was not covered by insurance.

Office systems. The third major subtheme was office systems. Participants stressed the importance of confidentiality of the medical record, confidential treatment of sensitive information by the staff (including sexual orientation), and private space to fill out history forms. They did not want their chief complaint written where it would be visible to anyone. They did not like being asked the same personal questions by multiple people, especially if the person's role in the patient's care was unclear. Participants elected not to disclose personal information, including sexual orientation, if they thought the chart was not confidential.

Another important issue was examination room comfort. Long waits, small examination gowns, gowns made of paper, and cold rooms make visits uncomfortable. Environmental factors clearly impacted women's healthcare experiences: "I don't like the time when you're in the exam room undressed, sitting there in this doily and waiting. You're looking at the stirrups; it's not a friendly sight at all. And it's usually cold."

\section{Patient-provider relationship}

The final major theme emerging from the analysis related to the patient-provider relationship. Comments were categorized as patient-provider relationship if they addressed attributes of the provider or characteristics of the provider's interactions with the patient. The three major subthemes identified were (1) communication style, (2) provider characteristics, including gender, sexual orientation, and knowledge, and (3) patients being known as individuals.

Communication style. The first subtheme was communication style. Participants highly valued solid communication skills, nonjudgmental attitudes, and nonhierarchical relationships. Women sought a provider who was willing to address difficult issues with full disclosure. Attentive listening was essential, as was accessible language: "I feel like they always want to fix things with med- 
icine first. And I would like someone who is willing to talk to me and figure out what my lifestyle is, and if other things can work first before I have to take pills."

Trust was a major component of ideal care. A major barrier to a trusting patient-provider relationship stemmed from the shaming behaviors of providers, which undermined good communication. Some women dreaded healthcare visits because of the focus on poor health habits. There was the sense that providers were constantly chastising patients about weight loss, exercise, smoking cessation, and drug or alcohol use, without much of an understanding of the underlying issues in an individual patient's life that contribute to these health behaviors: "'You should eat less and exercise more and lose weight.' If it was that easy everyone would do it, it's shaming. It's the same thing with the gowns, I'm not going to say 'This gown is too small.' Those things are hard, I just don't sit around my house eating Snickers, there is a host of other things going on."

In fact, some women delayed or avoided healthcare because of poor communication: "Ideally [healthcare] would be about me in my life, and not me in my life as the doctor perceives it. My doctor is not going to be my best friend, but awareness would be something huge. I think that's one of the reasons I don't seek healthcare now."

Participants were often reluctant to disclose their sexuality to providers because of the negative reaction they received or because the participants perceived that healthcare providers might consider homosexuality a mental illness: "When you verify, you say, 'I have sex with women,' they're like, 'Oh, okay, well, we're moving on.' I think it's true. I think they just want to move as quickly as possible away from the crazy lesbian." "There are a lot of doctors who still believe that being gay is a mental illness."

Most women had not been asked about sexual orientation, and they had experienced an assumption of heterosexuality from providers. This usually occurred in the context of reproductive health. Providers either repeatedly asked questions about the need for birth control or were concerned about pregnancy despite the patient's disclosure of sexual orientation. Providers' homophobia led some to drop out of treatment: "I wanted to see how they would react when I said I was a lesbian, and if I didn't get good vibes, I was out of there."
Communication skills, attitude, and knowledge in an ideal relationship were linked to specific issues facing lesbians. For example, the way in which a lesbian was asked (or not asked) about her sexual orientation strongly impacted the patient-provider relationship. Providers who had good communication skills and were nonjudgmental and accepting were favored, in part because their attitude invited disclosure of sexual orientation. Participants believed that if a provider asked about sexual orientation either directly or via questionnaire, it conveyed interest in and knowledge about what it meant to be lesbian: "One doctor I went to actually asked on the questionnaire, were you heterosexual, lesbian, gay, or bisexual. And that was wonderful. I could tell that they wouldn't be asking if they weren't open and aware. I thought, this tells them what my lifestyle is, and what my needs are, to a large extent."

Provider characteristics. The second subtheme within this theme was provider characteristics. Participants thought that women providers in general, and lesbians in particular, might have the attitudes and communication skills that were ideal and would be more attuned to the participant's experience: "I like to be sitting across from someone who is more like myself. I feel like they're a lot more in touch with the things that I might be prone to because of my ethnicity as well my sexual preference." However, they cited difficulty with access to lesbian providers: "I'd love to have a lesbian doctor, but on most of the insurance plans that I've had in the past, I've never had access to one."

Not all participants shared these sentiments. A number of women had had good experiences with male providers and nonlesbian providers. Overall, participants thought that the attitude and communication skills of the provider were most important. "To me, the gender or sexual orientation of the caregiver doesn't really matter as long as they treat me the way I think I should be treated, and as long as I feel that they have expertise." Knowledge, or expertise, was another provider characteristic considered ideal. Participants desired providers who were knowledgeable about gender differences in medical concerns, lifestyle choices such as vegetarianism, integrative therapies, and issues that predominantly affect women, such as domestic and sexual abuse: "Healthcare providers [should] have 
sort of a broad-based education about the lesbian lifestyle. This might sound basic but yes, lesbians do have children, some lesbians sleep with men occasionally. So, you can't say, they're lesbians so you don't ask them about these issues either."

The lack of evidence-based, health-related information specific to lesbians was cited as a barrier. Women called for more provider education and more research specific to lesbian health: "I know the information is not very clear because they haven't done a lot of research on woman-towoman transmission [of infectious diseases]." "With some providers, as the lesbian, you're educating them."

Knowing the patient as an individual. The third subtheme was that women wanted a provider who knew them as individuals. Knowledge of the patient's past history and the sense that the provider had read the chart was important. A provider who took a personal interest in the "whole person" and with whom a patient could have more of a "peer relationship" was discussed as ideal more often among the younger women: "I wish when I went to the doctor I was going for more than [pelvic examinations] because I've never really had a doctor that I wanted to talk to about my life, or my lifestyle choices, or anything like that, and I'm really just going because someone told me I needed a Pap smear every year."

Women found that providers assumed they were heterosexual, and this meant that they had to repeatedly disclose their sexual orientation: "But every single time [when asked about birth control needs] you have to explain, and you have to come out, it's like, here we go again." This led to avoidance of healthcare (to avoid repeated disclosure) or nondisclosure and perhaps unnecessary tests and examinations.

\section{DISCUSSION}

Our focus group analysis revealed that participants preferred care that is comprehensive in scope, person centered, and inclusive of them as lesbians; often their actual experiences were otherwise. Comprehensive care, as formulated by participants, follows the biopsychosocial model of treating the whole person ${ }^{27}$ and includes, or at least accepts as legitimate, integrative medicine practices. Women perceived care as inclusive of them as lesbians if it did not assume heterosexuality and was not discriminatory.

Comprehensive care and person-centered care emerged as interwoven ideas in our focus group analyses. Women favored integration of all aspects of their healthcare (including preventive, nutritional, acute, medical subspecialty, reproductive, gynecological, and mental healthcare). To deliver such comprehensive care, women stressed that providers must first acknowledge them as individuals (not widgets) and take the time to listen attentively to their specific needs. Unfortunately, women noted a gap between actual and ideal care; the actual care they received was often perceived as fragmented and shaming.

To our knowledge, the structure of healthcare delivery preferred by lesbians has not been explored previously. However, prior surveys of lesbians examining the doctor-patient relationship have identified a preference for providers who are compassionate and supportive, consistent with a biopsychosocial approach. ${ }^{28}$ This supports our finding that in terms of doctor-patient interactions, the ideal providers have excellent communication skills and approach their patients with a nonjudgmental, accepting attitude about a range of sensitive issues, including obesity, smoking, and sexual orientation. Whereas others have suggested that lesbians tend to prefer female providers, ${ }^{29}$ in our study, providers' communication attributes and holistic approach were more important to participants than provider gender. This is consistent with an emerging literature suggesting that, for many women, physician gender is not a dominant issue. ${ }^{30-32}$

The preferences and experiences of healthcare described by the participants were consistent with the documented preferences among women in general in that they experienced healthcare at the level of the model of care, the process of care, and the provider of care. Consistent with the findings reported by Anderson et al. ${ }^{22}$ of the National Study of Women, our findings suggest that women prefer healthcare that is integrated, patient centered, easy to access, confidential, and provided in a comfortable setting by a knowledgeable and attentive provider. Participants in our study experienced healthcare not only as women, however, but also as lesbians. Our results offer insight into the specific preferences of lesbians and the experiences that form them. To treat lesbian health simply as women's health would be 
to neglect important concerns raised by our participants and echoed in the lesbian health literature.

In general, the participants typically experienced healthcare as exclusive of them as lesbians and related this to heterosexism. Heterosexism is manifest in an implicit assumption of heterosexuality and in discriminatory treatment once lesbian orientation is disclosed. The concepts of assumed heterosexuality and discrimination against homosexuals are well described in the literature. ${ }^{15,28,33}$ However, our intensive qualitative approach allowed us to understand some of the determinants of lesbians' perception of heterosexism at the provider level, at the system level, and at the level of scientific inquiry. That is, participants operationalized the construct of heterosexism.

First, at the provider level, participants reported experiencing assumed heterosexuality: providers frame their history taking in heterosexual terms (e.g., asking about birth control use or possible pregnancy). Neutral questions including gay, lesbian, or bisexual orientation as response options were perceived as inclusive. Likewise, a trusting patient-provider relationship promoted disclosure of sexual orientation. Even after disclosing sexual orientation, participants described unintentional discrimination, stemming from providers' lack of knowledge about lesbian health issues (e.g., STD prevention, cancer risk), perhaps reflecting in part the relative paucity of clinically oriented reviews of these topics in the primary care literature. ${ }^{10}$ They also experienced or at least feared direct discrimination in the form of scorn ("that crazy lesbian") or remoteness (e.g., shifting abruptly to the next topic). As has been shown in other studies, these experiences led some women to avoid care, potentially jeopardizing their health. ${ }^{8}$ In contrast, the opportunity for disclosure and acceptance of sexual orientation led to a perception of better healthcare. Prior studies suggest that a strong patient-provider relationship can indeed lead to better healthcare outcomes. ${ }^{34-36}$

Second, participants experienced the entire healthcare delivery system as being heterosexist. Assumed heterosexuality was evident in waiting rooms lacking lesbian-specific health information and health forms often asking questions about marital status without an option for specifying lesbian orientation. Lesbians feared direct discrimi- nation if their sexual orientation was discovered by staff through chart review. Confidentiality of medical records was considered essential. In the current political climate where confidentiality of health information is receiving unprecedented scrutiny, 37,38 our findings clarify that the issue is of paramount importance to lesbians.

Third, on a global level, women identified the lack of scientific inquiry into questions specific to the heath needs of lesbians as devaluing them and compromising their health. Participants argued that this should be rectified through further research, consistent with the IOM's call for attention to this field. ${ }^{1}$

This study has several limitations. By design, a small sample was recruited from the Boston area, so results may not be generalizable to lesbians living in other geographic regions. Results may also not generalize to heterosexual women (especially the lesbian-specific findings), although some of the priorities of women in our sample mirror those of general populations of women participating in parallel studies. ${ }^{22,39} \mathrm{Re}-$ sults also may not be generalizable to elderly lesbians; the oldest participant in our study was 63 years old. Another limitation is that participants self-identified as lesbian; their responses may not represent the views of lesbians who would be uncomfortable disclosing their sexual orientation in any setting, even a confidential focus group.

This study also had several strengths. Given the dearth of information about lesbians' preferences for healthcare, ${ }^{1}$ the inductive nature of qualitative research ${ }^{40-42}$ commends it to this type of question. Unlike many studies of lesbians, our study successfully recruited a sample with diverse age range, race and ethnicity, and access to healthcare. This is consistent with the reality that lesbians come from all demographic and socioeconomic backgrounds. ${ }^{1}$

In conclusion, it is clear that although lesbians do not always disclose their sexual orientation to providers, they do seek healthcare and are likely part of most practices that include women. This has important implications for healthcare providers, for institutions, and for society. Healthcare providers should ask about sexual orientation in a neutral way. They should respond to a patient's disclosure of sexual orientation with an accepting, person-centered attitude and with evidence-based healthcare information that takes all aspects of the patient's health into account. Insti- 
tutions seeking to outreach to lesbians should develop systems of care that integrate primary care, mental healthcare, and integrative healthcare. Institutions should also ensure that all staff that interface with patients and their families approach sexual orientation sensitively and confidentially. Indeed, even the physical environment should be welcoming, with lesbian-specific pamphlets in the waiting room and health information forms inclusive of lesbians. At a societal level, more research is needed on lesbian-specific healthcare issues so that providers can be trained to deliver evidence-based care. Based on our participants' input, such measures would be expected to enhance the healthcare experience, and perhaps even healthcare outcomes, of lesbians.

\section{ACKNOWLEDGMENTS}

We gratefully acknowledge the technical expertise provided by Bonnie J. Sherman, Ph.D., and Valerie W. Jackson, B.A.

\section{REFERENCES}

1. Solarz A, ed. Institute of Medicine. Lesbian health: Current assessment and directions for the future. Washington, DC: National Academy Press, 1999.

2. Gonsiorek J, Weinrich J. Homosexuality: Research implications for public policy. Newbury Park, CA: Sage Publications, 1991.

3. Aaron DJ, Chang YF, Markovic N, LaPorte RE. Estimating the lesbian population: A capture-recapture approach. J Epidemiol Community Health 2003;57: 207.

4. Rankow EJ. Breast and cervical cancer among lesbians. Womens Health Issues 1995;5:123.

5. Diamant AL, Schuster MA, McGuigan K, Lever J. Lesbians' sexual history with men: Implications for taking a sexual history. Arch Intern Med 1999;159:2730.

6. Diamant AL, Schuster MA, Lever J. Receipt of preventive health care services by lesbians. Am J Prev Med 2000;19:141.

7. Diamant AL, Wold C, Spritzer K, Gelberg L. Health behaviors, health status, and access to and use of health care: A population-based study of lesbian, bisexual, and heterosexual women. Arch Fam Med 2000;9:1043.

8. Rankow E, Tessaro I. Cervical cancer risk and Papanicolaou screening in a sample of lesbian and bisexual women. J Fam Pract 1998;47:139.

9. Bradford J, Ryan C. The National Lesbian Health Care Survey: Final report. Washington, DC: National Lesbian and Gay Health Foundation, 1987.
10. White JC, Levinson W. Lesbian health care. What a primary care physician needs to know. West J Med 1995;162:463.

11. Abbott LJ. The use of alcohol by lesbians: A review and research agenda. Subst Use Misuse 1998;33:2647.

12. Johnson SR, Smith EM, Guenther SM. Comparison of gynecologic health care problems between lesbians and bisexual women. A survey of 2,345 women. J Reprod Med 1987;32:805.

13. Smith EM, Johnson SR, Guenther SM. Health care attitudes and experiences during gynecologic care among lesbians and bisexuals. Am J Public Health 1985;75:1085.

14. Carroll NM. Optimal gynecologic and obstetric care for lesbians. Obstet Gynecol 1999;93:611.

15. Trippet SE, Bain J. Physical health problems and concerns of lesbians. Women Health 1993;20:59.

16. Johnson SR, Guenther SM, Laube DW, Keettel WC. Factors influencing lesbian gynecologic care: A preliminary study. Am J Obstet Gynecol 1981;140:20.

17. White JC, Dull VT. Health risk factors and health-seeking behavior in lesbians. J Womens Health 1997;6:103.

18. Asbury J. Overview of focus group research. Qual Health Res 1995;5:414.

19. Morgan DL. Why things (sometimes) go wrong in focus groups. Qual Health Res 1995;5:516.

20. Scholle SH, Weisman CS, Anderson R, Weitz T, Freund KM, Binko J. Women's satisfaction with primary care: A new measurement effort from the PHS National Centers of Excellence in Women's Health. Womens Health Issues 2000;10:1.

21. Poses RM, Isen AM. Qualitative research in medicine and health care: Questions and controversy. J Gen Intern Med 1998;13:32.

22. Anderson RT, Barbara AM, Weisman C, et al. A qualitative analysis of women's satisfaction with primary care from a panel of focus groups in the National Centers of Excellence in Women's Health. J Womens Health Gend Based Med 2001;10:637.

23. Denzin N, Lincoln Y. Handbook of qualitative research, 2nd ed. Thousand Oaks, CA: Sage Publications, 2000.

24. Glaser B, Strauss A. The discovery of grounded theory: The strategies for qualitative research. Hawthorne, NY: Aldine Transaction, 1967.

25. Ryan GW, Bernard HR. Techniques to identify themes. Field Methods 2003;15:85.

26. Waitzkin H. On studying the discourse of medical encounters. A critique of quantitative and qualitative methods and a proposal for reasonable compromise. Med Care 1990;28:473.

27. Engel GL. The need for a new medical model: A challenge for biomedicine. Science 1977;196:129.

28. Stevens PE. Lesbians' health-related experiences of care and noncare. West J Nurs Res 1994;16:639.

29. Zeidenstein L. Gynecological and childbearing needs of lesbians. J Nurse Midwifery 1990;35:10.

30. Plunkett BA, Kohli P, Milad MP. The importance of physician gender in the selection of an obstetrician or a gynecologist. Am J Obstet Gynecol 2002;186:926. 
31. Fisher WA, Bryan A, Dervaitis KL, Silcox J, Kohn H. It ain't necessarily so: Most women do not strongly prefer female obstetrician-gynaecologists. J Obstet Gynaecol Can 2002;24:885.

32. Howell EA, Gardiner B, Concato J. Do women prefer female obstetricians? Obstet Gynecol 2002;99:1031.

33. Stevens PE, Hall JM. Stigma, health beliefs and experiences with health care in lesbian women. Image J Nurs Sch 1988;20:69.

34. Safran DG, Taira DA, Rogers WH, Kosinski M, Ware JE, Tarlov AR. Linking primary care performance to outcomes of care. J Fam Pract 1998;47:213.

35. Kaplan SH, Greenfield S, Ware JE Jr. Assessing the effects of physician-patient interactions on the outcomes of chronic disease [published erratum appears in Med Care 1989;27:679]. Med Care 1989;27:S110.

36. McLeod JR. Accepting homosexuality. Can Fam Physician 1994;40:1505.

37. Welch CA. Sacred secrets-The privacy of medical records. N Engl J Med 2001;345:371.

38. Annas GJ. A national bill of patients' rights. N Engl J Med 1998;338:695.
39. Bean-Mayberry BA, Chang CC, McNeil MA, Whittle J, Hayes PM, Scholle SH. Patient satisfaction in women's clinics versus traditional primary care clinics in the Veterans Administration. J Gen Intern Med 2003; 18:175.

40. Stevens PE. Focus groups: Collecting aggregate-level data to understand community health phenomena. Public Health Nurs 1996;13:170.

41. Mishler EG. Research interviewing: Context and narrative. Cambridge, MA: Harvard University Press, 1991.

42. Berkwits M, Inui TS. Making use of qualitative research techniques. J Gen Intern Med 1998;13:195.

Address reprint requests to: Margaret R. Seaver, M.D., M.P.H. $V A$ Boston Healthcare System 150 S. Huntington Avenue (11PCC/WHC) Boston, MA 02130

E-mail: margrsea@massmed.org 